\title{
LEVEL CROSSINGS OF A RANDOM TRIGONOMETRIC POLYNOMIAL WITH DEPENDENT COEFFICIENTS
}

\author{
K. FARAHMAND \\ (Received 7 June 1991; revised 10 March 1992)
}

Communicated by Tony Pakes

\begin{abstract}
This paper provides an asymptotic estimate for the expected number of $K$-level crossings of the random trigonometric polynomial $g_{1} \cos x+g_{2} \cos 2 x+\ldots+g_{n} \cos n x$ where $g_{j}(j=1,2, \ldots, n)$ are dependent normally distributed random variables with mean zero and variance one. The two cases of $\rho_{j r}$, the correlation coeffiecient between the $j$-th and $r$-th coefficients, being either (i) constant, or (ii) $\rho^{|j-r|}$ $j \neq r, 0<\rho<1$, are considered. It is shown that the previous result for $\rho_{j r}=0$ still remains valid for both cases.
\end{abstract}

1991 Mathematics subject classification (Amer. Math. Soc.): primary 60G99; secondary 42BXX. Keywords and phrases: number of real roots, Kac-Rice formula, random trigonometric polynomial.

\section{Introduction}

Suppose that $g_{1}(\omega), g_{2}(\omega), \ldots, g_{n}(\omega)$ is a sequence of random variables defined on a probability space $(\Omega, A, P)$, each normally distributed with mathematical expectation zero and variance one, and that $N_{n, K}(a, b) \equiv N(a, b)$ is the number of real roots of the equation $T(\theta)=K$ where

$$
T(\theta) \equiv T_{n}(\theta, \omega)=\sum_{j=1}^{n} g_{j}(\omega) \cos j \theta .
$$

Dunnage [2] has shown that in the case of independent coefficients in the interval $0 \leq \theta \leq 2 \pi$ all save a certain exceptional set of equations $T(\theta)=0$ have $2 n / \sqrt{3}+$ $O\left\{n^{11 / 13}(\log n)^{3 / 13}\right\}$ roots, when $n$ is large. The measure of his exceptional set does not exceed $(\log n)^{-1}$. For $K \neq 0$ such that $K=o(\sqrt{n})$ Farahmand [3] and [4] has shown that the mathematical expectation of $N(0,2 \pi)$, denoted by $E N(0,2 \pi)$, is

(C) 1995 Australian Mathematical Society 0263-6115/95 \$A2.00+0.00 
asymptotic to $2 n / \sqrt{3}$. Here we consider the effect of coefficients being dependent on $E N(a, b)$. We show that the above asymptotic formula persists whenever the correlation coefficient between any two coefficients $g_{j}$ and $g_{r}$, denoted by $\rho_{j r}$ is either (i) constant, or (ii) $\rho^{|j-r|} j \neq r, 0<\rho<1$. For the case of $K=0$ the same result has been obtained separately by Sambandham [8] and Renganathan and Sambandham [5]. We prove the following results in this paper.

THEOREM 1. If the coefficients of $T(x)$ in (1.1) are normally distributed random variables with mean zero, variance one and $\rho_{j r}$, the correlation coefficients between $j$-th and $r$-th coefficients, are either (i) constant, or (ii) $\rho^{|j-r|} j \neq r, 0<\rho<1$, then for all sufficiently large $n$ and any constant $K$, the expected number of real roots of the equation $T(\theta)=K$ satisfies

$$
E N(0,2 \pi)=2 n / \sqrt{3}+O\left(n^{3 / 4}\right) \quad \text { if } K=O\left(n^{3 / 8}\right)
$$

and

$$
E N(0,2 \pi)=2 n / \sqrt{3}+o(n) \quad \text { if } K=o(\sqrt{n}) .
$$

\section{Preliminary Analysis}

Let

$$
A^{2}=\sum_{j=1}^{n} \cos ^{2} j \theta, \quad B^{2}=\sum_{j=1}^{n} j^{2} \sin ^{2} j \theta
$$

and

$$
C=\sum_{j=1}^{n} j \sin j \theta \cos j \theta .
$$

By using the expected number of level crossings given by Cramér and Leadbetter [ 1 , page 285] for the equation $T(\theta)-K$ we can obtain

$E N(a, b)=\int_{a}^{b} \frac{\Delta^{1 / 2}}{\alpha} \phi\left(-\frac{K}{\alpha^{1 / 2}}\right)\left[\phi\left(\frac{K \gamma}{\alpha^{1 / 2} \Delta^{1 / 2}}\right)+K \gamma\left\{2 \Phi\left(\frac{K \gamma}{\alpha^{1 / 2} \Delta^{1 / 2}}\right)-1\right\}\right] d \theta$

where

$$
\begin{aligned}
& \alpha=\operatorname{var}\{T(\theta)\}=A^{2}+\sum_{j \neq r}^{n} \sum_{j}^{n} \rho_{j r} \cos j \theta \cos r \theta, \\
& \beta=\operatorname{var}\left\{T^{\prime}(\theta)\right\}=B^{2}+\sum_{j \neq r} \sum_{j r} j r \cos j \theta \sin r \theta, \\
& \gamma=\operatorname{Cov}\left\{T(\theta), T^{\prime}(\theta)\right\}=-C-\sum \sum_{j \neq r} \rho_{j r} j \cos j \theta \sin r \theta,
\end{aligned}
$$




$$
\begin{aligned}
& \Delta=\alpha \beta-\gamma^{2}, \quad \Phi(t)=(2 \pi)^{-1 / 2} \int_{-\infty}^{t} \exp \left(-y^{2} / 2\right) d y \quad \text { and } \\
& \phi=\Phi^{\prime}(t)=(2 \pi)^{-1 / 2} \exp \left(-t^{2} / 2\right) .
\end{aligned}
$$

From (2.1) and since $\Phi(t)=1 / 2+(\pi)^{-1 / 2} \operatorname{erf}(t / \sqrt{2})$ we have the extension of the Kac-Rice formula [5]

$$
\begin{aligned}
E N(a, b)= & \int_{a}^{b}\left(\Delta^{1 / 2} / \pi \alpha\right) \exp \left(-K^{2} \beta / 2 \Delta\right) d \theta \\
& \quad+(\sqrt{2} / \pi) \int_{a}^{b}|K \gamma| \alpha^{-3 / 2} \exp \left(-K^{2} / 2 \alpha\right) \operatorname{erf}\left(|k \gamma| / \sqrt{2} \alpha^{1 / 2} \Delta^{1 / 2}\right) d \theta \\
(2.5) \quad= & I_{1}(a, b)+I_{2}(a, b),
\end{aligned}
$$

say.

Let $S(\theta)=\sin (2 n+1) \theta / \sin \theta$ then the terms $A^{2}, B^{2}$ and $C$ appearing in (2.5) can all be written as a function of $S(\theta)$ as follows. Since

$$
S(\theta)=1+2 \sum_{j=1}^{n} \cos 2 j \theta
$$

we have

$$
A^{2}=(1 / 2) \sum_{j=1}^{n}(1+\cos 2 j \theta)=n / 2+(1 / 4)\{S(\theta)-1\} .
$$

Also since from (2.6)

$$
S^{\prime \prime}(\theta)=-8 \sum_{j=1}^{n} j^{2} \cos 2 j \theta=4 \sum_{j=1}^{n} j^{2}\left(2 \sin ^{2} j \theta-1\right),
$$

we have

$$
B^{2}=n(n+1)(2 n+1) / 12+(1 / 8) S^{\prime \prime}(\theta) .
$$

From (2.7) we also obtain

$$
C=(-1 / 2) \frac{d}{d \theta}\left(A^{2}\right)=(-1 / 8) S^{\prime}(\theta) .
$$

As they will be required later, we define

$$
D=\sum_{j=1}^{n} \cos j \theta \quad \text { and } \quad E=\sum_{j=1}^{n} j \sin j \theta
$$

From (2.6) we can show that

$$
D=(1 / 2)\{S(\theta / 2)-1\}
$$


and

$$
E=(-1 / 4) S^{\prime}(\theta) .
$$

As $S(\theta)$ occurs frequently, we collect together some related inequalities. From (2.6) it is obvious that as $n \rightarrow \infty$,

$$
S^{(v)}(\theta)=O\left(n^{\nu+1}\right)
$$

uniformly in $\theta$. Stricter inequalities can be obtained by confining $\theta$ to the intervals $\epsilon \leq \theta \leq \pi-\epsilon$ and $1+\epsilon \leq \theta \leq 2 \pi-\epsilon$, where $\epsilon$ is any positive constant smaller than $\pi$. Then, since $|S(\theta)|<1 / \sin \epsilon$, we can obtain

$$
S(\theta)=O(1 / \epsilon)
$$

Also

$$
S^{\prime}(\theta)=(2 n+1) \cos (2 n+1) \theta / \sin \theta-\cot \theta S(\theta)=O(n / \epsilon)
$$

and

$$
\begin{aligned}
S^{\prime \prime}(\theta) & =-(2 n+1)^{2} S(\theta)-(2 n+1) \cos \theta \cos (2 n+1) \theta \sin ^{-2} \theta \\
& =O\left(n^{2} / \epsilon\right) .
\end{aligned}
$$

These together with $(2.7)-(2.10)$ give

$$
\begin{aligned}
A^{2} & =n / 2+O(1 / \epsilon), \\
B^{2} & =n^{3} / 6+O\left(n^{2} / \epsilon\right), \\
C & =O(n / \epsilon), \\
D & =O(1 / \epsilon) \quad \text { and } \\
E & =O(n / \epsilon) .
\end{aligned}
$$

\section{Proof of the Theorem}

We shall divide the roots of $T(\theta)-K=0$ into two groups: (i) those lying in the intervals $(0, \epsilon),(\pi-\epsilon, \pi+\epsilon)$ and $(2 \pi-\epsilon, 2 \pi)$, and (ii) those lying in the intervals $(\epsilon, \pi-\epsilon)$ and $(\pi+\epsilon, 2 \pi-\epsilon)$. For the roots of type (i) which, it so happens, are negligible, we need some modification to apply Dunnage's [2] approach. Those roots which make a significant contribution to the final result are of type (ii) and their expected number is found by using the Kac-Rice formula (2.5). The choice of $\epsilon$ is important. It must not be too large, so that we can deal easily with type (i) roots; but 
if it is too small the approximation for type (ii) will become inadequate. We will see $\epsilon=n^{-1 / 4}$ is sufficient for both requirements.

First we consider the case of $\rho_{j r} \equiv \rho$ (constant). From (2.2), (2.11) and (2.14) We have

$$
\alpha=A^{2}+\rho \sum_{j \neq r}^{n} \sum^{n} \cos j \theta \cos r \theta=A^{2}+\rho\left\{\left(\sum_{j=1} \cos j \theta\right)^{2}+\sum_{j=1}^{n} \cos ^{2} j \theta\right\}
$$

(3.1) $=(1-\rho) A^{2}+\rho D^{2}=n(1-\rho) / 2+O\left(\epsilon^{-2}\right)$.

Similarly from (2.3), (2.4) and (2.12) we can obtain

$$
\begin{aligned}
& \beta=n^{3}(1-\rho) / 6+O\left(n^{2} \epsilon^{-2}\right), \quad \text { and } \\
& \gamma=O(n \epsilon-2) .
\end{aligned}
$$

Hence from (3.1)-(3.3) we have

$$
\Delta=n^{4}(1-\rho)^{2} / 12+O\left(n^{3} \epsilon^{-2}\right) .
$$

So from (2.5) and (3.1)-(3.4) we can write

$$
I_{1}(\epsilon, \pi-\epsilon)=(n \sqrt{3})\{1+O(\epsilon)\} \exp \left\{-2 K^{2} / n(1-\rho)+O\left(K^{2} / n^{2} \epsilon^{2}\right)\right\}
$$

and

$$
I_{2}(\epsilon, \pi-\epsilon)=O\left(K^{3} n^{-3 / 2} \epsilon^{-2}\right) .
$$

Hence for $K=O\left(n^{3 / 8}\right)$ from (2.5), (3.5) and (3.6) we have

$$
E N(\epsilon, \pi-\epsilon)=n / \sqrt{3}+O\left(n^{3 / 4}\right),
$$

and for $K=o(\sqrt{n})$,

$$
E N(\epsilon, \pi-\epsilon)=n / \sqrt{3}+o(\sqrt{n}) .
$$

Now we turn to the intervals $(0, \epsilon),(\pi-\epsilon, \pi+\epsilon)$ and $(2 \pi-\epsilon, 2 \pi)$, and we show that the equation has a negligible expected number of real roots in these intervals. By periodicity, the expected number of real roots in $(0, \epsilon)$ and $(2 \pi-\epsilon, 2 \pi)$ is the same as the expected number in $(-\epsilon, \epsilon)$. We shall therefore confine ourselves to this last interval; the interval $(\pi-\epsilon, \pi+\epsilon)$ can be treated in exactly the same way to give the same result. The idea, due to Dunnage [2], is to consider the random integral function $T(z, \omega)-K$ of the complex variable $z$. The number of real roots between $\pm \epsilon$ does not exceed the number in the circle $|z|<\epsilon$. Let $N(r) \equiv N(r, \omega, K)$ denote 
the number of real roots of $T(z, \omega)-K=0$ in $|z|<r$. Assuming that $T(0) \neq K$ then by Jensen's theorem [9, page 125] or [7, page 332] we have

$$
N(\epsilon) \log 2 \leq(2 \pi)^{-1} \int_{0}^{2 \pi} \log \mid\left\{T\left(2 \epsilon e^{i \theta}, \omega\right)-K\right\} /\{T(0)-K\} d \theta .
$$

Let $\Lambda^{2}=n+\rho n(n-1)$; then, by standard probability theory, the distribution function of $T(O, \omega)=\sum_{j=1}^{n} g_{j}(\omega)$ is

$$
G(x)=\left(2 n \Lambda^{2}\right)^{-1 / 2} \int_{-\infty}^{x} \exp \left(-t^{2} / 2 \Lambda^{2}\right) d t,
$$

from which, for any positive $v$, we can see that $|T(0, \omega)-K|>e^{-v}$ except for sample functions in an $\omega$-set of measure not exceeding

$$
\left(2 \pi \Lambda^{2}\right)^{-1 / 2} \int_{K-e^{-\nu}}^{K+e^{-\nu}} \exp \left(-t^{2} / 2 \Lambda^{2}\right) d t<2\left(2 \pi \Lambda^{2}\right)^{-1 / 2} e^{-\nu} .
$$

Also since $\left|\cos \left(2 n \epsilon e^{i \theta}\right)\right| \leq 2 e^{2 n \epsilon}$ we have

$$
\left|T\left(2 \epsilon e^{i \theta}\right)\right| \leq 2 e^{2 n \epsilon}\left(\left|g_{1}\right|+\left|g_{2}\right|+\ldots+\left|g_{n}\right|\right) \leq 2 n e^{2 n \epsilon} \max \left|g_{j}\right|
$$

where the maximum is taken over $1 \leq j \leq n$. The distribution function of $\left|g_{j}\right|$ is

$$
F(x)= \begin{cases}\sqrt{2 / \pi} \int_{0}^{x} \exp \left(-t^{2} / 2\right) d t & x \geq 0 \\ 0 & x<0 .\end{cases}
$$

Now if $\max \left|g_{j}\right|>n e^{v}$ then $\left|g_{j}\right|>n e^{\nu}$ for at least one value of $j \leq n$, so that

$$
\begin{aligned}
\operatorname{Prob}\left(\max \left|g_{j}\right|>n e^{\nu}\right) & \leq \sum_{j=1}^{n} \operatorname{Prob}\left(\left|g_{j}\right|>n e^{\nu}\right) \leq n \operatorname{Prob}\left(\left|g_{1}\right|>n e^{\nu}\right) \\
& =n \sqrt{2 / \pi} \int_{n e^{\nu}}^{\infty} \exp \left(-t^{2} / 2\right) d t \sim \sqrt{2 / \pi} \exp \left(-v-n^{2} e^{2 \nu} / 2\right)
\end{aligned}
$$

for all sufficiently large $n$. Therefore from (3.11) and (3.12) except for sample functions in an $\omega$-set of measure not exceeding $(2 / \pi)^{1 / 2} \exp \left(-v-n^{2} e^{2 v} / 2\right)$,

$$
\left|T\left(2 \epsilon e^{i \theta}\right)-K\right|<\left|n^{2} \exp (2 n \epsilon+v)-K\right| .
$$

Combining (3.10) with (3.13) and since for both $K=O\left(n^{3 / 8}\right)$ and $K=o(\sqrt{n})$

$$
\left|n^{2} \exp (2 n \epsilon+v)-K\right|<2 n^{2} \exp (2 n \epsilon+v)
$$


for all $\theta$ we get

$$
\left|\left\{T\left(2 \epsilon e^{i \theta}, \omega\right)-K\right\} /\{T(0, \omega)-K\}\right|<2 n^{2} \exp (2 n \epsilon+2 \nu)
$$

except for sample functions in an $\omega$-set of measure not exceeding

$$
2\left(2 \pi \Lambda^{2}\right)^{-1 / 2} e^{-v}+(2 / \pi)^{1 / 2} \exp \left(-v-n^{2} e^{2 \nu} / 2\right)
$$

Therefore from (3.9) and (3.14) we can show that outside the exceptional set

$$
N(\epsilon) \leq(\log 2+2 \log n+2 n \epsilon+2 \nu) / \log 2 .
$$

Since $\epsilon=n^{-1 / 4}$ from (3.15) and for all sufficiently large $n$

$$
\begin{aligned}
\operatorname{Prob}\{N(\epsilon)>3 n \epsilon+2 \nu\}< & 2\left(2 \pi \Lambda^{2}\right)^{-1 / 2} e^{-\nu} \\
& +(2 / \pi)^{1 / 2} \exp \left(-\nu-n^{2} e^{2 \nu} / 2\right) .
\end{aligned}
$$

Let $n^{\prime}$ be the integer part of $3 \sqrt{n}$. Then from (3.16) and for $n$ sufficiently large we have

$$
\begin{aligned}
E N(\epsilon) & =\sum_{j>0} \operatorname{Prob}\{N(\epsilon) \geq j\} \\
& =\sum_{1 \leq j \leq n^{\prime}} \operatorname{Prob}\{N(\epsilon)>j\}+\sum_{j \geq 1} \operatorname{Prob}\left\{N(\epsilon)>n^{\prime}+j\right\} \\
& \leq n^{\prime}+2\left(2 \pi \Lambda^{2}\right)^{-1 / 2} \sum_{j \geq 1} e^{-j / 2}+(2 / \pi)^{1 / 2} \sum_{j \geq 1} \exp \left\{-j / 2-n^{2} e^{j} / 2\right\} \\
& =O\left(n^{3 / 4}\right) .
\end{aligned}
$$

This gives an upper bound for the number of real roots of $T(\theta)$ in the interval $(-\epsilon, \epsilon)$, which together with (3.6) and (3.7) completes proof of the theorem for the case of $\rho_{j r} \equiv \rho$.

Now we consider the case of $\rho_{j r}=\rho^{|j-r|}, j \neq r, 0<\rho<1$. To avoid repetition we only point out the adjustment necessary in the calculation of $\alpha, \beta, \gamma$. For $\epsilon<0<\pi-\epsilon$ from (2.14) we have

$$
\sum_{j \neq r} \sum^{|j-r|} \cos j \theta \cos r \theta \leq D^{2}=O\left(\epsilon^{-2}\right)
$$

which together with (2.2) and (2.11) gives

$$
\alpha=A^{2}+\sum_{j \neq r} \sum^{|j-r|} \cos j \theta \cos r \theta=n / 2+O\left(\epsilon^{-2}\right) .
$$


Similarly, since from (2.14) and (2.15)

$$
\sum_{j \neq r} j r \rho^{|j-r|} \sin j \theta \sin r \theta<E^{2}=O\left(n^{2} \epsilon^{-2}\right)
$$

and

$$
\sum_{j \neq r} j \rho^{|j-r|} \sin \theta \sin r \theta<D E=O\left(n \epsilon^{-2}\right)
$$

we obtain

$$
\beta=n^{3} / 6+O\left(n^{2} \epsilon^{-2}\right)
$$

and

$$
\gamma=O\left(n^{2} \epsilon^{-2}\right)
$$

Now (3.18)-(3.20) are sufficient for obtaining (3.7) and (3.8). For the intervals $(0, \epsilon),(\pi-\epsilon, \pi+\epsilon)$ and $(2 \pi-\epsilon, 2 \pi)$ the same argument remains valid if we replace $A^{2}$ in (3.10) by

$$
\Lambda^{\prime 2}=n+\sum_{j \neq r} \rho^{|j-r|}
$$

Then since $\Lambda^{\prime 2}<\Lambda^{2}$ from (3.17) we can obtain $E N(\epsilon)$, which completes the proof of the theorem for the case $\rho_{j r}=\rho^{|j-r|}$.

\section{References}

[1] H. Cramér and M. R. Leadbetter, Stationary and related stochastic processes (Wiley, New York, 1967).

[2] J. E. A. Dunnage, 'The number of real zeros of a random trigonometric polynomial', Proc. London Math. Soc. 16 (1966), 53-84.

[3] K. Farahmand, 'On the average number of level crossings of a random polynomial', Ann. Probab. 18 (1990), 1403-1409.

[4] - 'Level crossings of a random trigonometric polynomial', Proc. Amer. Math. Soc. 111 (1991), $551-557$.

[5] N. Renganathan and M. Sambandham, 'On the average number of real zeros of a tandom trigonometric polynomial with dependent coefficients', Indian J. Pure Appl. Math. 15 (1984), 951-956.

[6] S. O. Rice, 'Mathematical theory of random noise', Bell System Tech. 25 (1945), 46-156.

[7] W. Rudin, Real and complex analysis, 2nd edition (McGraw-Hill, New York, 1974).

[8] M. Sambandham, 'On random trigonometric polynomial', Indian J. Pure Appl. Math. 7 (1976), 841-849.

[9] E. C. Titchmarsh, The theory of functions, 2nd edition (Oxford University Press, Oxford, 1939).

Department of Mathematics

University of Ulster

Jordanstown

Co Antrim

BT37 OQB

United Kingdom 